\title{
Roberto POLITo (éd.), Aenesidemus of Cnossus. Testimonia
}

Mauro Bonazzi

\section{OpenEdition}

Journals

Édition électronique

URL : https://journals.openedition.org/philosant/469

DOI : 10.4000/philosant.469

ISSN : 2648-2789

\section{Éditeur}

Éditions Vrin

\section{Édition imprimée}

Date de publication : 24 novembre 2015

Pagination : 280-282

ISBN : 978-2-7574-1141-4

ISSN : $1634-4561$

Référence électronique

Mauro Bonazzi, «Roberto poцıтo (éd.), Aenesidemus of Cnossus. Testimonia », Philosophie antique [En ligne], 15 | 2015, mis en ligne le 01 novembre 2018, consulté le 03 décembre 2022. URL : http:// journals.openedition.org/philosant/469; DOI : https://doi.org/10.4000/philosant.469

\section{(ब) $\Theta \Theta$}

Creative Commons - Attribution - Pas d'Utilisation Commerciale - Pas de Modification 4.0 International - CC BY-NC-ND 4.0

https://creativecommons.org/licenses/by-nc-nd/4.0/ 
qui prédomine dans un mélange), pour penser la multiplicité des degrés selon un schéma de continuité analogique (p. 343).

O. Gilon («Vertus cardinales et théologales chez saint Augustin », p. 363388) revient sur le double héritage - hellénistique et paulinien - du saint Docteur. Les vertus ne peuvent se réaliser sans le secours de la grâce divine. L'indépendance de la volonté par rapport à l'intelligence signifie que la connaissance ne suffit pas à fonder la vertu (p. 373). Augustin s'écarte encore de la philosophie ancienne en admettant des étapes intermédiaires entre l'absence de sagesse et son acquisition, mais aussi en niant que la pleine possession de la vertu soit accessible ici-bas.

Le volume se clôt sur un article de M. Dixsaut, «Deux éthiques aristocratiques » (p.389-417), qui rapproche deux perspectives traditionnellement opposées, celles de Platon et de Nietzsche qui partagent une « conception aristocratique de la vertu » (p.416) : le projet de perfectionner l'humain par la promotion d'un certain « type » d'homme, la dénonciation de la falsification des valeurs, l'invention d'une nouvelle culture/éducation sont autant de thèmes autour desquels se retrouvent les deux penseurs.

L'ouvrage comprend une conclusion (p.419-434), une riche bibliographie (p. 435-452), un index locorum (p. 453-471) et un index nominum des auteurs modernes (p. 473-476). Les quatorze études réunies ici proposent des approches détaillées et souvent originales sur les différents traitements qu'a pu recevoir la question. On pourrait toutefois s'étonner de ce qu'aucune ne soit consacrée à l'aspect socratique et aux dialogues (Protagoras, Ménon) qui l'initient. La conclusion propose néanmoins quelques pistes encore à explorer sur ce sujet (p. 419-420), prouvant que, bien qu'abondamment discutée, la question de l'unité des vertus demeure un champ d'investigation stimulant.

Laetitia MONTEILS-LAENG

Université de Montréal

Roberto Polito (éd.), Aenesidemus of Cnossus. Testimonia, Cambridge, Cambridge University Press, 2014 (Cambridge Classical Texts and Commentaries, 52), ISBN 9780521190251.

L'importance d'une édition des témoignages d'Énésidème s'impose de toute évidence pour tous ceux qui s'occupent du scepticisme grec. Le problème est bien connu: le pyrrhonisme constitue un des mouvements philosophiques les plus intéressants de l'Antiquité et son influence a traversé les siècles jusqu'aujourd'hui. Mais qu'est-ce que le pyrrhonisme, quels sont ses origines et son développement ? Nous sommes informés sur les deux extrêmes de cette histoire, Pyrrhon et Sextus Empiricus. Mais Pyrrhon n'était pas pyrrhonien: son indifférentisme n'a pas beaucoup à faire avec la philosophie du scepticisme; Sextus Empiricus, le chapitre conclusif du pyrrhonisme antique, est en revanche un sceptique avéré, mais il semble être plutôt un rapporteur qu'un penseur original. L'édition de R. Polito nous permet enfin de concentrer notre attention sur le penseur qui réside au 
milieu et qui, selon toute probabilité, a été l'«inventeur»du scepticisme pyrrhonien, Énésidème de Cnossos. L'importance d'Énésidème était évidemment déjà reconnue par tous le spécialistes; mais disposer enfin d'un recueil ordonné et systématique des témoignages le concernant donnera une très grande impulsion à l'étude de sa pensée.

Après une brève introduction, qui nous présente les sources principales (Photius, Sextus Empiricus, Diogène Laërce, Aristoclès de Messène, Tertullien), le livre se divise en deux sections, la première et la plus courte consacrée aux données historiques, la seconde, et la plus importante, à la pensée de notre auteur. En suivant l'exemple d'autres éditions, chaque témoignage (l'auteur n'estimant pas utile de distinguer entre fragments proprement dits et témoignages) présente le texte grec avec la traduction, suivi par une overview et un commentary on selected words or sentences. Les overviews sont souvent très longues, et constituent de fait de petits essays sur les sujets les plus importants. Ce choix est très utile, et compense, au moins en partie, l'absence d'une présentation d'ensemble de l'interprétation d'Énésidème développée par l'auteur (chose très commune dans les éditions de fragments).

Les témoignages sur Énésidème ne sont pas tous clairs, ce qui explique le grand nombre de débats parmi les spécialistes. Il est impossible de rendre compte de tous ces problèmes. Mais parler de certains d'entre eux pourra aider à comprendre à la fois l'intérêt de la pensée d'Énésidème et en quoi consiste l'originalité de la présente édition. D'un point de vue historique, une question importante concerne l'affiliation académicienne prétendue d'Énésidème. Longtemps considérée comme allant de soi, elle a été contestée par Fernanda Decleva Caizzi avant d'être défendue par Jaap Mansfeld. Polito conforte, à l'aide de remarques très pertinentes, l'hypothèse de Fernanda Caizzi : même en laissant de côté les problèmes lexicaux (comme par exemple le sens de hairesis et synairesiotes), il semble en effet peu vraisemblable qu'Énésidème ait pu souligner son lien avec les académiciens dans un ouvrage qui contenait une polémique très dure contre le pseudo-scepticisme de l'Académie hellénistique (p.44). Un second problème, lié au premier, porte sur l'interprétation de Platon qu'Énésidème aurait developpée. Sextus en parle dans la section finale du premier livre des Esquisses pyrrhoniennes, consacrée à la confrontation entre le pyrrhonisme et les autres philosophies (les parakeimenai philosophiai, c'est-à-dire les philosophies qui peuvent être rapprochées du pyrrhonisme). Mais le texte est corrompu et ne permet pas d'établir clairement si Énésidème avait défendu ou attaqué la possibilité d'un lien entre le scepticisme et Platon (I 222223). Selon Polito, qui à mon avis voit juste, Énésidème aurait nié que Platon ait pu être considéré comme compatible avec le pyrrhonisme. Sur ce point, qui est strictement lié à la polémique contre l'Académie hellénistique, Sextus est donc d'accord avec lui. (À ce propos, il vaut la peine de remarquer que Polito [p. 157] adopte la correction proposée par Emidio Spinelli [ « Sextus Empiricus, the Neighbouring Philosophies and the Sceptical Tradition » dans J. Sihvola (éd.), Ancient 
Scepticism and the Sceptical Tradition, Helsinki, 2000, p. 39], selon laquelle Sextus nie que Platon soit sceptique kataper hoi peri Menodoton kai Ainesidemon, « as do those who follow Menodotus and Aenesidemus ». Philologiquement, la correction est très plausible et aide à rendre le texte plus clair et cohérent.) En revanche, là où Énésidème et Sextus divergent, c'est sur Héraclite, mais Polito a raison de souligner que la divergence ne concerne pas la nature du pyrrhonisme en lui-même, mais simplement la compatibilité d'Héraclite avec le pyrrhonisme : pour Sextus, le langage utilisé par Héraclite est dogmatique et donc Héraclite est incompatible avec le scepticisme ; pour Énésidème, la thèse héraclitéenne du conflit des expériences va en revanche dans la même direction que le pyrrhonisme, en dépit du langage employé (p. 295-298).

Il ne s'agit pas, bien entendu, de simple érudition. Tout au contraire, ces points nous montrent un aspect original de l'interprétation de Polito. Le milieu d'incubation du scepticisme pyrrhonien d'Énésidème n'est pas l'Académie hellénistique mais plutôt la médecine empirique (qu'Énésidème aurait pu connaître à Alexandrie; voir p. 8, 54-57 à propos d'Héraclide de Tarente). Voilà une hypothèse très suggestive, que les spécialistes du pyrrhonisme sont invités à explorer dans les prochaines années. Plus précisement, l'intérêt d'une telle hypothèse est lié à un concept fondamental du pyrrhonisme à l'époque impériale, à savoir le concept de phainomenon. S'il est bien connu que l'appropriation et la transformation de Pyrrhon en sceptique passe par cette notion, le problème qui demeure est celui d'établir quelle est la valeur précise de phainomenon, et si elle est la même pendant toute l'histoire du pyrrhonisme (en d'autres mots, d'un point de vue plus proprement historique, il s'agit d'établir encore une fois le degré de compatibilité entre Énésidème et Sextus). En effet, on pourrait observer qu' « apparaître » se dit en plusieurs sens; en général ce verbe peut indiquer ce qui apparaît à partir de l'expérience sensible, mais il peut aussi se référer au contenu de tous nos jugements. Il est intéressant de remarquer avec Polito qu'Énésidème semble exploiter la notion de phainomenon dans le premier sens seulement, à la différence de Sextus mais comme les médecins empiristes (p. 139-152). Ce parallèle semble constituer une preuve très importante de l'incubation du scepticisme pyrrhonien dans le champ de la médecine empirique (une autre piste intéressante, toujours à propos de ce problème, est celle qui conduit aux cyrénaïques, voir en particulier p. 152; et voir aussi p. 290 à propos de l'évaluation positive du plaisir). En outre, il montre aussi d'une façon exemplaire l'intérêt et l'importance de cette édition : dans la mesure où elle nous aide à comprendre que l'histoire du pyrrhonisme est plus compliquée et moins linéaire qu'on ne le pense habituellement, on peut s'attendre à ce qu'elle donne une nouvelle impulsion à l'étude du pyrrhonisme et, plus généralement parlant, du scepticisme, dans sa richesse et sa complexité.

Mauro BONAZZI

Université de Milan 